Article

\title{
The Influence of Honed Surfaces on Metal-on-Metal Hip Joints
}

\author{
Dipankar Choudhury ${ }^{1)^{*}}$, Robert Walker ${ }^{2)}$, Ayoub Shirvani ${ }^{2)}$ and Rajshree Mootanah ${ }^{2)}$ \\ ${ }^{1)}$ Department of Biomedical Engineering, University of Malaya \\ 50603 Kuala Lumpur, Malaysia \\ ${ }^{2)}$ Medical Engineering Research Group, Faculty of Science and Technology, Anglia Ruskin University \\ Chelmsford, UK \\ *Corresponding author: dipankar.choudhury78@gmail.com
}

( Manuscript received 13 December 2012; accepted 21 March 2013; published 30 April 2013 )

\begin{abstract}
Wear rate and debris are key problems to implanted metal-on-metal hip joints. Surface texture on bearing surfaces is reported to increase tribological performances. Research on the translation of this technology to metal-on-metal hip joint for reduction of friction, wear rate and debris generation is limited. The aim of this study was to investigate, by theoretical predictions and experimental investigations, the tribological properties of simulated metal-on-metal hip joints with different surface textures. Three different honed surfaces were produced with emery paper at controlled load and speed. The experiments were carried out using a computer-controlled friction simulator. Theoretical prediction was carried out using an existing model for textured surfaces. Both experimental and theoretical results demonstrated that honed surfaces had lower friction coefficients during walking and stairs ascent and descent, demonstrating their potential use in metal on metal hip joints for increased implant longevity.
\end{abstract}

Keywords: honed surface, texture, friction, hydrodynamic lubrication, hip joint implant

\section{Introduction}

Metal-on-metal (MoM) hip prostheses have become more popular than conventional metal-on-polyethylene (MoP) hip prostheses because of their low wear rates. Ceramic-on-ceramic (CoC) hip prostheses have lower friction coefficients and wear rates, but are brittle. The percentage of hip replacements performed with MoM hip prostheses was 14\% in England and Wales [1] and 35\% in the USA [1]. However, the durability of MoM implants, biological reaction of metallic wear debris with the surrounding tissues and toxic metal deposit in blood have been significant challenges [1-3]. The UK National Joint Registry [1] reported 8,309 revision procedures between 2008 and 2010 in England alone.

Textured bearing surfaces produce lower wear rates than non-textured surfaces due to reduced contact surface areas, reduced friction coefficients and removal of wear debris, which further reduce surface wear [4-8]. The use of honed surface in the engine cylinder has been effective in reducing friction and wear rate, thereby increasing the engine life span. Therefore, the honed surface technique could potentially be translated to MoM hip joint surfaces to reduce friction and wear rate. However, MoM hip joint surfaces operate at a lower range of speed compared to engine piston and cylinder surfaces. The relative speed between the bearing surfaces is a key element to generate thicker hydrodynamic fluid film $[9,10]$, which could help separate the matching surfaces, increase load bearing capacity, and reduce friction coefficient and wear rate $[7,8,11,12]$. Although the honed surface technique has been successful, there are very few examples of its application in MoM hip joints surfaces. Gao et al.'s numerical study on the effect of surface texturing on MoM hip implants [13] predicted that surface texturing could help improve the tribological performance of asperity contact ratio and lubrication performance of MoM hip replacement. Ito et al. [14] showed that a well-designed surface texture in MoP hip joints resulted in a significant reduction in percentage of friction $(30 \%)$ and wear rate $(68 \%)$ after $10 \times 10^{5}$ cycles. However, to our knowledge, no study has investigated the effect of the plateau honed surface technique on a MoM hip joint bearing. The aim of this study was to investigate, by laboratory investigations and theoretical analysis, the effect of honed surface textures on the tribological properties of MoM hip joints.

\section{Study procedures}

The aims of the laboratory investigations were to manufacture simulated hip joints with different bearing 
surface profiles and to identify the profile corresponding to the lowest friction coefficient. A theoretical prediction was then performed to validate these experimental findings. Simulated artificial hip joints were manufactured from cobalt-chromium-molybdenum (Cr-Co-Mo) because of its extensive use in orthopedic implants, given its excellent biocompatibility and hardness properties [15]. To simulate the different surface textures, a set of plane surfaces $(n=6)$ and three sets of honed surfaces $(n=4,6,6)$ were produced, using different grades of emery paper under controlled applied

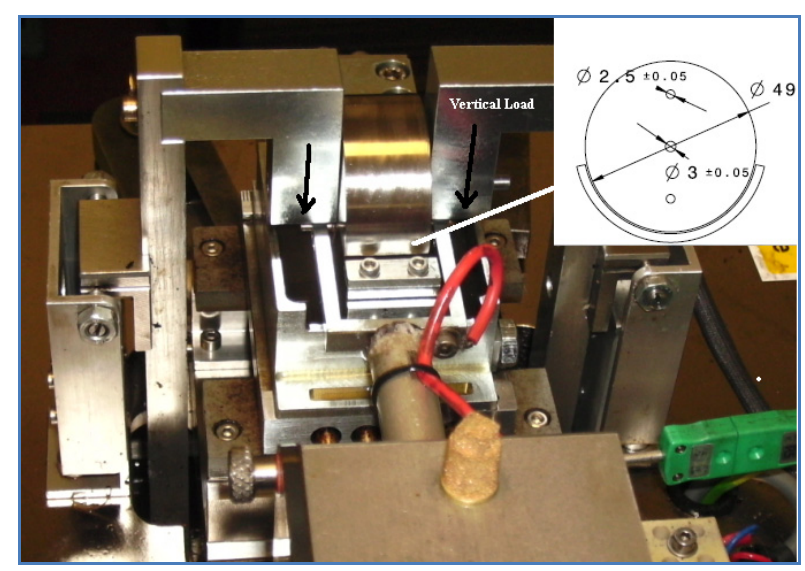

Fig. 1 Experimental specimen (dimensions are in $\mathrm{mm})$ loading conditions. Surface roughnesses $(R a)$ of these four surface profiles were measured, using a Taylor-Hobson Surtronic $3^{+}$machine (Taylor Hobson Ltd, Leicester, UK). The number of grooves $(m)$, their widths $\left(l_{d}\right)$, and distances between two successive grooves $(l)$ were measured, using a scanning electron microscope (Camscan, Cambridge, UK). Groove heights $\left(h_{d}\right)$ were measured, using a Talysurf CCI (Taylor Hobson Ltd, UK). The mean and standard deviation values of these parameters were then determined, using SPSS 16 software (SPSS Inc, USA).

\subsection{Experimental study}

The specimens were prepared with $50 \mathrm{~mm}$ diameter Cr-Co-Mo cylinders and sleeves of $5 \mathrm{~mm}$ long, with a 50 $\mu \mathrm{m}$ interface clearance, which is shown in the Fig. 1. This dimension was chosen because a ' $50 \mathrm{~mm}$ diameter and $50 \mu \mathrm{m}$ interface clearance' was reported to be the best geometrical combination for MoM hip joints [16,17]. Dynamic loading was applied according to published gait data [18] and simulator capacity. Contact forces and relative speed in a hip joint vary with type of gaits, as shown in Table 2. As the contact area of the experimental surface (approximately $196 \mathrm{~mm}^{2}$ ) is different from the contact area of a $50 \mathrm{~mm}$ MoM hip joint ( approximately $1962.5 \mathrm{~mm}^{2}$ ), the load was calculated by the area ratio of 10 for the contact surface of a specimen. A TE77 friction test simulator (Phoenix Tribology Ltd, England), consisting of the COMPEND 2000 software and a control unit with a Serial Link Interface Module (SLIM)

Table 1 Average surface roughness $(R a)$ and parameters of plane and honed surfaces

\begin{tabular}{c|c|c|c|c|c}
\hline \multicolumn{7}{c}{ Surface parameters } \\
\hline Surface types & $\begin{array}{c}\text { Surface } \\
\text { Roughness } R a \\
\text { before tests }(\mu \mathrm{m})\end{array}$ & $\begin{array}{c}\text { Honed } \\
\text { depth } \\
h_{\mathrm{d}},(\mu \mathrm{m})\end{array}$ & $\begin{array}{c}\text { Honed } \\
\text { width } \\
l_{\mathrm{d},}(\mu \mathrm{m})\end{array}$ & $\begin{array}{c}\text { Distance } \\
\text { between two } \\
\text { textures } l(\mu \mathrm{m})\end{array}$ & $\begin{array}{c}\text { Number of } \\
\text { textures } m\end{array}$ \\
\hline Plane surface & $3.34 \pm 0.5$ & - & - & - & - \\
\hline Honed surface 1 & $3.38 \pm 0.5$ & $10 \pm 1$ & $16 \pm 5$ & 295 & 68 \\
\hline Honed surface 2 & $3.4 \pm 0.5$ & $12 \pm 1$ & $20 \pm 5$ & 300 & 52 \\
\hline Honed surface 3 & $3.6 \pm 0.5$ & $17 \pm 1.5$ & $40 \pm 5$ & 375 & 68 \\
\hline
\end{tabular}

Table 2 Applied load and speed of specimen used in the experiment

\begin{tabular}{|c|c|c|c|c|}
\hline Activity & $\begin{array}{c}\text { Experimental } \\
\text { Speed } \\
(\mathrm{m} / \mathrm{sec})\end{array}$ & Actual Load & $\begin{array}{c}\text { Experimental Load } \\
(\mathrm{N})\end{array}$ & $\begin{array}{c}\text { Experimental maximum } \\
\text { Hertz pressure (MPa) }\end{array}$ \\
\hline Medium walks & 0.59 & 1500 & 150 & 38.4 \\
\hline Fast walks & 0.65 & 1550 & 155 & 39.0 \\
\hline Slow walks & 0.55 & 1480 & 148 & 38.1 \\
\hline Stair ascends & 0.46 & 1640 & 164 & 40.2 \\
\hline Stair descends & 0.44 & 1750 & 175 & 41.5 \\
\hline
\end{tabular}




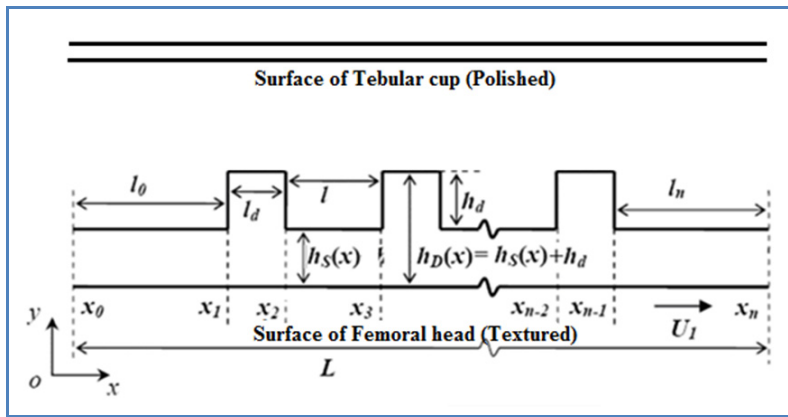

Fig. 2 Two-dimensional geometry of surface texture (theoretical)

2000, connected to a host PC provided automatic sequence control and data recording every second. The experiments were performed at a controlled temperature of $37^{\circ} \mathrm{C}$ [19], to simulate human body temperature. In this experiment, Morris Multilife synthetic $(5 \mathrm{~W} / 30)$ lubricant was used because it had a viscosity of $70 \mathrm{cP}$ at $37^{\circ} \mathrm{C}$, which was similar to those of pseudo-synovial fluid, and had a similar working capability over a long-term experiment. As it was a synthetic lubricant, it exhibited greater stability of viscosity with higher loads, which is similar to synovial fluid. The corresponding average coefficients of friction of the four different surface profiles were calculated and compared after the first twelve hundred cycles to identify the honed surface profile that resulted in minimum friction coefficient, which is associated with increased longevity of MoM hip replacements. Wear was only inspected from surface condition before and after the test.

\subsection{Theoretical prediction study}

Theoretical values of friction coefficients for the different honed surfaces were predicted, employing a published theoretical model [20] that used a set of two-dimensional equations for different predetermined dimpled surface parameters and relative velocity of bearing surfaces. Dimple and honed surfaces can be geometrically identical in a two-dimensional profile, as shown in Fig. 2. Friction coefficients for the honed surfaces in this study could therefore be predicted, using Rahmani's derived equations in the theoretical model $[20,21]$ to better understand tribological factors of honed surfaces in metallic hip joints.

Following on Fig. 2, Frictional force $\left(F_{f}\right)$ and bearing loading capacity $(W)$ can be expressed

$$
F_{f}=-\sum_{i=1}^{n}\left[4 \mu U \frac{\left(x_{i}-x_{i-1}\right)}{h_{i}}+c_{1} \frac{\left(x_{i}-x_{i-1}\right)}{2 h_{i}^{2}}\right]
$$

$$
\begin{array}{r}
W=6 \mu U \sum_{i=1}^{n}\left[\begin{array}{l}
\frac{\left(x_{i}-x_{i-1}\right)^{2}}{2 h_{i}^{2}} \\
\left.+\left(x_{i}-x_{i-1}\right) \sum_{j=1}^{i-1} \frac{\left(x_{j}-x_{j-1}\right)}{h_{j}^{2}}\right]
\end{array}\right] \\
+c_{1} \sum_{i=1}^{n}\left[\begin{array}{l}
\frac{\left(x_{i}-x_{i-1}\right)^{2}}{2 h_{i}^{3}} \\
+\left(x_{i}-x_{i-1}\right) \sum_{j=1}^{i-1} \frac{\left(x_{j}-x_{j-1}\right)}{h_{j}{ }^{3}}
\end{array}\right]+c_{2} \sum_{i=1}^{n}\left(x_{i}-x_{i-1}\right)
\end{array}
$$

Theoretical coefficient of friction, $\eta=\frac{F_{f}}{W}$

Where $c_{1}$ and $c_{2}$ are the integrating constants and $c_{l}$ can be calculated from the equations:

$$
c_{1}=\frac{\left(P_{x_{n}}-P_{x_{0}}\right)-6 \mu U \sum_{i=1}^{n} \frac{\left(X_{i}-X_{i-1}\right)}{h_{i}^{2}}}{\sum_{i=1}^{n} \frac{\left(X_{i}-X_{i-1}\right)}{h_{i}^{3}}}
$$

But $P_{x_{n}}=P_{x_{0}}=P_{a t m}=$ atmospheric pressure and at atmospheric pressure $c_{2}=0$

$$
\text { Therefore, } c_{1}=\frac{-6 \mu U \sum_{i=1}^{n} \frac{\left(x_{i}-x_{i-1}\right)}{h_{i}^{2}}}{\sum_{i=1}^{n} \frac{\left(x_{i}-x_{i-1}\right)}{h_{i}^{3}}}
$$

The equations can be rearranged as follows [21]

$$
\begin{aligned}
F_{f}= & -\left(4 \mu U \frac{l_{0}}{h_{s}}+c_{1} \frac{l_{0}}{2 h_{s}^{2}}\right)-m\left(4 \mu U \frac{l_{d}}{h_{d}}+c_{1} \frac{l_{d}}{2 h_{d}^{2}}\right) \\
& -(m-1)\left(3 \mu U \frac{l}{h_{s}}+c_{1} \frac{l}{2 h_{s}^{2}}\right)-\left(3 \mu U \frac{l_{n}}{h_{s}}+c_{1} \frac{l_{n}}{2 h_{s}^{2}}\right) \\
W= & 6 \mu U\left[\frac{l_{0}^{2}}{2 h_{s}^{2}}+m l_{d} \frac{l_{0}}{h_{s}^{2}}+(m-1) l\left(\frac{l_{0}}{h_{s}^{2}}\right)+\frac{l_{n}^{2}}{2 h_{s}^{2}}\right. \\
& +l_{n}\left(\frac{l_{0}}{h_{s}^{2}}\right)+m l_{n} \frac{l_{d}}{h_{d}^{2}}+(m-1) l_{n}\left(\frac{l}{h_{s}^{2}}\right) \\
& +\frac{m^{2}}{2}\left(\frac{l_{d}^{2}}{h_{d}^{2}}\right)+\frac{m(m-1)}{2} l\left(\frac{l_{d}}{h_{d}^{2}}\right) \\
& \left.+\frac{m(m-1)}{2} l_{d}\left(\frac{l}{h_{s}^{2}}\right)+\frac{(m-1)^{2}}{2}\left(\frac{l^{2}}{h_{s}^{2}}\right)\right] \\
& +c_{1}\left[\frac{l_{0}^{2}}{2 h_{s}^{3}}+m l_{d} \frac{l_{0}}{h_{s}^{3}}+(m-1) l\left(\frac{l_{0}}{h_{s}^{3}}\right)\right. \\
& +\frac{l_{n}^{2}}{2 h_{s}^{3}}+l_{n}\left(\frac{l_{0}}{h_{s}^{3}}\right)+m l_{n}\left(\frac{l_{d}}{h_{d}^{3}}\right) \\
& +(m-1) l_{n}\left(\frac{l}{h_{s}^{3}}\right)+\frac{m^{2}}{2}\left(\frac{l_{d}^{2}}{h_{d}^{3}}\right)+\frac{m(m-1)}{2} l\left(\frac{l_{d}}{h_{d}^{3}}\right) \\
& \left.+\frac{m(m-1)}{2} l_{d}\left(\frac{l}{h_{s}^{3}}\right)+\frac{(m-1)^{2}}{2}\left(\frac{l^{2}}{h_{s}^{3}}\right)\right] \\
& \left.\frac{m}{2}\right) \\
& \left.\frac{m}{2}\right)
\end{aligned}
$$


While, $c_{1}=\frac{-6 \mu U\left[\frac{l_{0}}{h_{s}^{2}}+m \frac{l_{d}}{h_{d}^{2}}+(m-1) \frac{l}{h_{s}^{2}}+\frac{l_{n}}{h_{s}^{2}}\right]}{\left[\frac{l_{0}}{h_{s}^{3}}+m \frac{l_{d}}{h_{d}^{3}}+(m-1) \frac{l}{h_{s}^{3}}+\frac{l_{n}}{h_{s}^{3}}\right]}$

The measured groove numbers $(m)$, their widths $\left(l_{d}\right)$ and heights $\left(h_{d}\right)$, and distances between two successive grooves $(l)$ were the input parameters in the theoretical study (Adapted from the Table 1). Minimum film thicknesses $\left(h_{s}\right)$ for the simulated walking and stairs ascent and descent were calculated, using the Hamrock-Dowson Formula [22].

$$
h_{\mathrm{s}}=2.798 R_{x}\left(\frac{\mu U}{E R_{x}}\right)^{0.65}\left(\frac{L}{E R_{x}}\right)^{-0.21}
$$

The equivalent cylinder radius value $\left(R_{x}\right)$ [23] was 25 $\mathrm{mm}$ (radius of experiment specimen) and the equivalent elastic modulus $(E)$ for Chromium cobalt was $220 \mathrm{GPa}$ [13]. These two values remained the same for the different activities since the material and dimension of the specimen remained unchanged. The theoretical coefficient of friction $(\eta)$ for the different surfaces during simulated walking and stairs ascent and descent were calculated, using MathCAD Prime 1.0 professional software (PTC, Needham, USA), after inputting texture and specimen parameters $\left(R_{x}, E\right)$ in the equations derived from equations 6,7 and 8 [20,21].

\section{Results and analyses}

\subsection{Laboratory investigation}

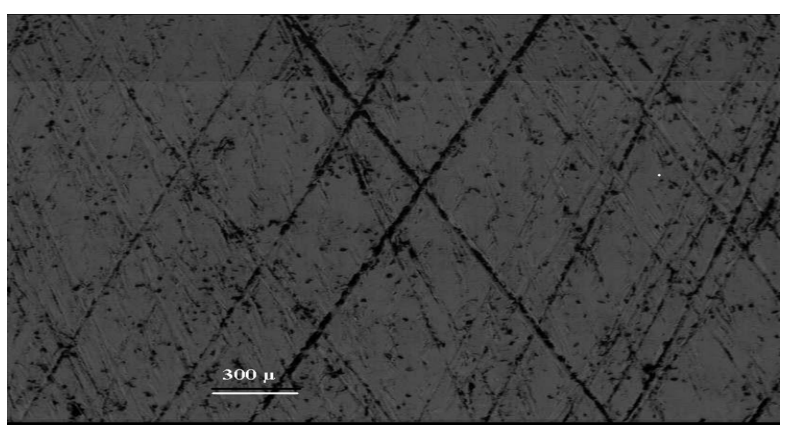

(a)

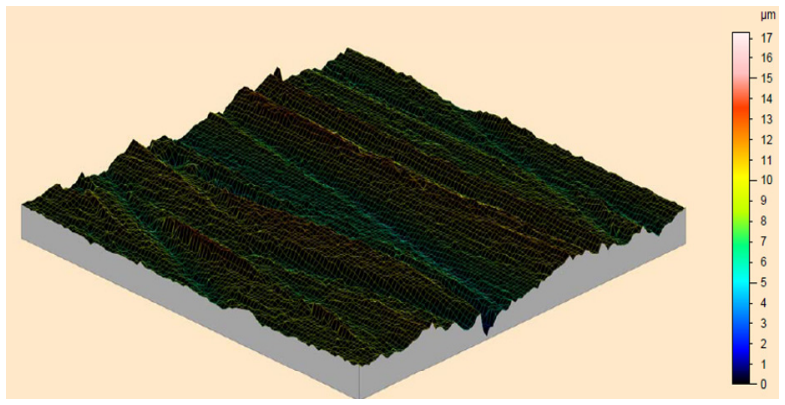

(b)

Fig. 3 Honed surface 3: a) Under SEM b) Under Talysurf CCI
The mean and standard deviation (SD) values of the plane and honed surface profile parameters (Roughness, depths, widths, number of and distances between honed profiles and groove heights are presented in Table 1. A representative surface profile for honed surface 3 is shown in Fig. 3.

Fig. 4 presents the friction coefficients $(\eta)$ of simulated MoM hip joints with plane and different honed surface profiles, subjected to medium walking speed at $37^{\circ} \mathrm{C}$. Polished surfaces $(n=6)$ produced higher friction coefficients compared to the honed surfaces. Honed surface $3(n=6)$ had the lowest friction coefficient among the honed surfaces. Although one honed surface 1 sample had a very low friction coefficient of 0.04 , the rest of samples of surface 1 had consistent results of 0.046 (0.0017). Fig. 4 illustrates that honed surfaces 2 had the highest friction coefficient values of 0.054 (0.001) and honed surface 3 had the least friction coefficient values of $0.043(0.0028)$ in most cases (samples 2-6). Friction coefficient values varied within samples as a result of manufacturing defaults, higher surface roughness and the effect of wear debris. However,

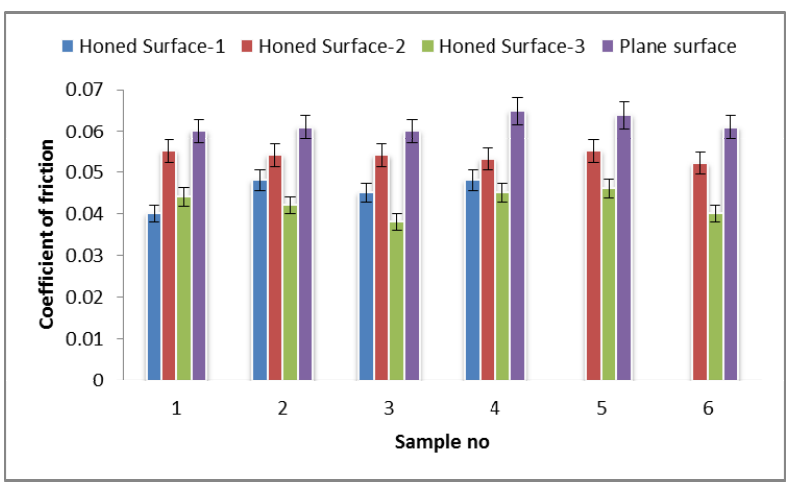

Fig. 4 Experimental coefficient of friction $(\eta)$ for different honed and plane surfaces with medium-speed walking

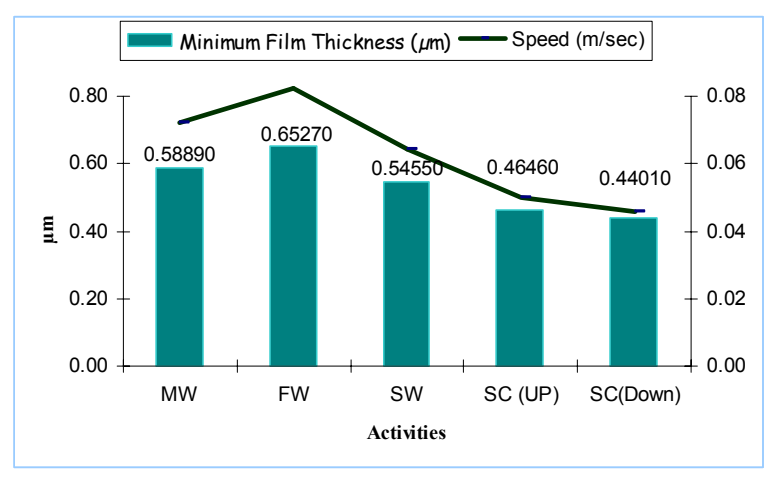

Fig. 5 Minimum film thickness generation with different gaits (MW=medium walking, $\mathrm{FW}=$ fast walking, $\mathrm{SW}=$ slow walking, $\mathrm{SC}($ Down $)=$ stairs descent, $\mathrm{SC}(\mathrm{UP})=$ stairs ascent) 
the key finding is that honed surface 3 had a significantly lower friction coefficient compared with honed surfaces 1 and 2.

\subsection{Theoretical simulation}

The minimum film thicknesses for simulated walking and stairs ascent and descent, predicted by Hamrock-Dowson Formula [24], are presented in Fig. 5. Again, friction coefficients for these gaits, projected by the Rahmani derived equations [20,21]. Honed surface 3 had the lowest friction coefficient $(0.0363)$, followed by the honed surfaces $1(0.0401)$ and $2(0.0423)$. Honed surface 3 consisted of a set of deeper and wider honed grooves but fewer in number $(m)$, reflecting the lowest associated friction coefficient value. Although honed surface 1 had smaller groove width and depth and a higher number of grooves compared to honed surface 2 , its friction coefficient was higher. Therefore, the relationship between groove depth, width, number, and distance between two parallel grooves was non-linear for predicting friction coefficients. Fig. 6 presents the friction coefficients, obtained by theoretical and experimental methods, for honed surfaces 2 and 3 during simulated walking and stair ascent and descent. In both cases, honed surface 3 had a lower friction coefficient profile during the different activities. The predicted friction coefficient profiles were lower than experimental ones for every activity. Simulated faster speed walking resulted in higher relative speed between the simulated hip joint components. This is due to the generation of a thicker hydrodynamic fluid film that separates the bearing surfaces and the resulting lower friction coefficient, especially at speeds higher than 0.4 $\mathrm{m} / \mathrm{s}[4,9]$. Indeed, all experimental investigations and theoretical predictions showed that fast walking resulted in lower friction coefficient values compared with medium and slow walking speeds $\left(\eta_{S W}>\eta_{M W}>\eta_{F W}\right)$. However, the friction coefficient profiles were different for stairs ascent and descent. Theoretically predicted friction coefficients for stairs ascent $(0.043$ for honed surface 3$)$, were higher than those of walking ( 0.036 for

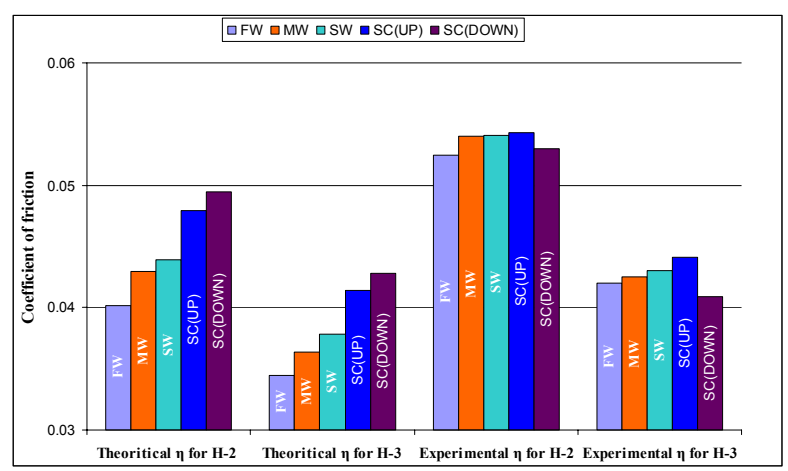

Fig. 6 Friction coefficient comparison for different activities (combination of honed surface 2 and 3) medium walking, 0.034 for fast walking and 0.038 for slow walking). However, experimental results show lower friction coefficient for stair ascent $(0.0409$ for honed surface 3$)$ than walking $(0.0425$ for medium walking, 0.0420 for fast walking and 0.0430 for slow walking), as illustrated in Fig. 6. This is because the predicted friction coefficients were based on the surface parameters, bearing speed, viscosity of the lubricant and bearing loading capacity. However, the experimental friction coefficient values were dependent on surface parameters, bearing speed, viscosity of the lubricant and normal load. Normal load was determined by body weight and simulator capacity, whereas predicted bearing loading capacity (603.88 $\mathrm{N}$ for honed surface 3 with medium speed walking) was calculated from surface parameters and speed which differed from the applied load of $148 \mathrm{~N}$ for simulated medium speed walking. This was due to the loading capacity limitation of the simulator. For example, during stairs ascent and descent, a hip joint bearing is usually subjected to a lower speed but higher applied load compared to walking. However, this applied load was not included in the theoretical bearing capacity equations. Classical theory also states that, at high pressure, friction force increases at lower velocities [25]. Moreover, it is found that applied load is a key factor in determining film thickness and friction coefficient [26]. However, the key finding is that honed surface 3 had lower friction

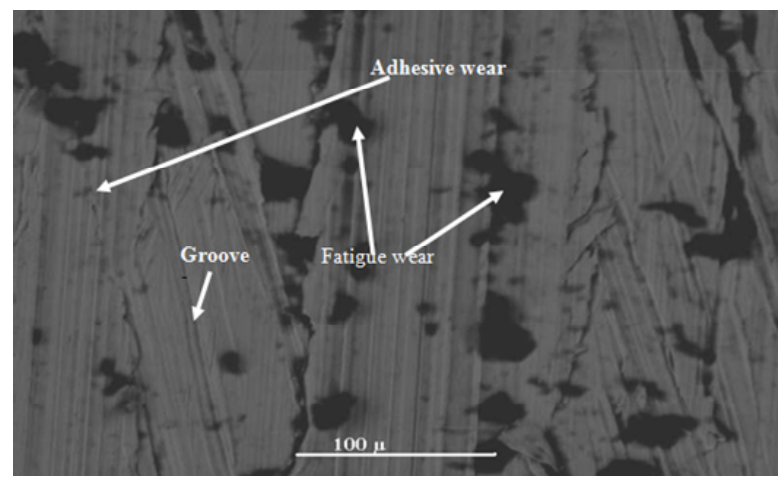

(a)

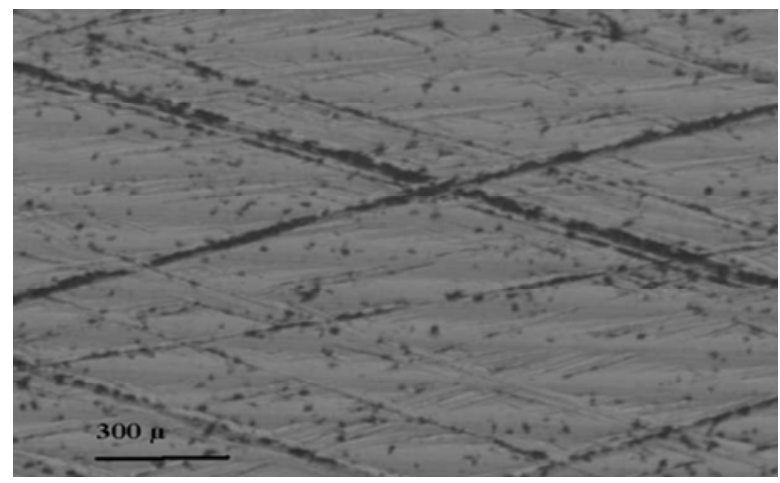

(b)

Fig. 7 (a) honed surface 2 and (b) honed surface 3 after the test 
coefficients for walking and stairs ascent and descent in both experimental and theoretically-predicted investigations. This could be because the combination of surface parameters (hone depth, width and number of grooves) of honed surface 3 were more appropriate for the MoM joints condition compared to honed surfaces 1 and 2.

\section{Discussion}

The mean surface roughness of the plane surface in this study $(R a 3.34 \pm 0.5 \mu \mathrm{m})$ was much higher than the standard surface roughness of commercial MoM hip joints $(R a 0.50 \pm 0.010 \mu \mathrm{m})[16,17]$. This is one of the main limitations of the study. Indeed, it is difficult to polish the surface of the cylinder and sleeve to a high standard. But, yet the cylindrical shaped specimen was chosen because it was the only option to fabricate a honed surface profile by using a conventional honing method which included a lath machine, different graded emery papers and a controllable loading device. However, all the honed surface profiles were based on the same profile of the plane surface $(R a 3.34 \pm 0.5 \mu \mathrm{m})$, and the honed surface profiles were at least three times deeper than the plane surface roughness (Table 1). Therefore, the relative friction coefficient values assumed not to be effected. Moreover, loading conditions, such as speed: $0.07 \mathrm{~m} / \mathrm{sec}$ (MW), normal load: $150 \mathrm{~N}$ (MW), temperature: $37^{\circ} \mathrm{C}$ (body temperature), matched those of the simulated MoM hip joint [27]. For example, Normal load of this study was $150 \mathrm{~N}$ for MW, which appears low compared to the physiological load applied on of the implanted MoM hip. However, if we convert into Hertz contact pressure (31.4 $\mathrm{MPa}$; appendix 1$)$, it remains within the ranges of Hertz pressure of simulated MoM hip joints (14-60 MPa) [27]. Mischler et al [27] calculated the ranges of Hertz pressure (14-60 MPa) from a metal analysis of experimental studies of CoCrMo alloys using tribometers and hip simulators. Chiba et al [28] also used similar range of Hertz pressure in their experiment which was contacted on carbon cast and low carbon forged Co-29Cr-6Mo alloys (MoM).

Theoretically-predicted friction coefficient values for walking activities had a similar pattern $\left(\eta_{F W}>\eta_{M W}>\right.$ $\left.\eta_{S W}\right)$ to those obtained experimentally. Note that the applied loads to these walking gaits differed by only \pm $4.6 \%$. On the other hand, friction coefficient patterns for stairs ascent were different (theoretical: $\eta_{S C(U P)}<$ $\eta_{S C(D O W N)}$ and experiment: $\eta_{S C(U P)}>\eta_{S C(D O W N)}$, where applied load differed by $\pm 17 \%$ [23]. The magnitudes of predicted and experimental friction coefficient profiles were different. For example, in case of medium speed walking, friction coefficient values differed by $14 \%$ for honed surface 1 and $22 \%$ for honed surface 2 . The reasons for these variations were:

1) The theoretical friction coefficient was predicted from bearing load carrying capacity $(603.88 \mathrm{~N}$ for honed surface 3 with medium speed walking), whereas the experimental friction coefficient was derived from the applied load (150 $\mathrm{N}$ to all honed surfaces with medium speed walking), which was a compromise between gait data [18] and the simulator capacity [23].

2) Factors such as wear debris, surface roughness, machining error (data record, setting the position of bearing surfaces), lubrication conditions and geometry of bearing surfaces affected the experimental results. Wear debris can act as third body abrasive wear and increase friction coefficients $[14,29,30]$. Surface roughness also results in high friction coefficient $[9,15,28,29]$. These factors were not included in the theoretical analysis, where the honed surfaces were considered ideal, without any manufacturing limitations. However, both experimental and theoretical results agreed with other published articles [8,13,14,27-29]. Rahmani et al. [20] calculated the optimum theoretical friction coefficient (0.001967) which was much lower than the theoretically-predicted results (lowest 0.036). It is to be noted that the calculated medium walking speed of $0.0722 \mathrm{~m} / \mathrm{s}$ was lower than engine piston cylinder bearing speed of $1 \mathrm{~m} / \mathrm{s}$ [20]. The optimum predicted height ratio $\left(h_{d} / h_{s}\right)$ from Rahmani's study was 1.57 [20], while that for honed surface 3 was 29.91. However, the friction coefficient values obtained experimentally agreed with those of Mezghani et al. [8] and Ito et al. [14], who recommended that the friction coefficient of a commercial bearing varied between 0.05 and 0.1 with textured surface. Sawano et al. [29] demonstrated that the durability of artificial joints could be extended to approximately 35 years by using a micro-dimpled surface with a set of $1 \mu \mathrm{m}$ deep dimple. Similarly, other experimental studies $[14,27]$ proved that surface texture could reduce friction coefficient and wear rate at a significant rate for simulated artificial hip joints. Friction coefficient values obtained by theoretical prediction (0.036-0.042) were similar with those obtained experimentally (0.043-0.054) in this study, and matched previously-published data $[13,14,30,31]$.

This comparison study serves as a validation for our experimental study. However, our study was only limited to the early phase of friction coefficient for honed surfaces. Nevertheless, this study presented a theoretical explanation for the superior tribological properties of honing surface 3 in our simulated MoM hip joint. Therefore, this analysis can be used to identify theoretically-optimised honed surface parameters (depth, width and number of grooves) for MoM joint bearing and verified by experimental studies to help improve the tribological performance of MoM hip joints. Moreover, this study shows that further work is warranted to identify the optimised honed parameters to minimise wear and friction coefficient, which should be conducted with high standard polished spherical 
specimens, and simulated body fluid as lubricant.

\section{Conclusion}

Both experimental and theoretical simulations encouraged the scope of applying honed surface techniques in MoM hip joint surfaces. Experimental results for all investigated activities showed that honed surface 3 gave a better tribological performances (friction coefficient and surface conditions) than honed surfaces 1 and 2. Theoretically-predicted results also showed better tribological performance (friction coefficient) of the honed surface 3 compared to the honed surfaces 1 and 2 . Therefore, the surface textured parameters with depth $=17 \pm 1.5 \mu \mathrm{m}$, width $=40 \pm 5 \mu \mathrm{m}$ and distance between two textures $=375 \mu \mathrm{m}$ resulted in lowest friction coefficient, which is associated with reduced wear, increased MoM hip implant longevity.

\section{Acknowledgements}

The research was supported by Anglia Ruskin University, UK and University of Malaya (Project no. RG147-12AET), Malaysia. The authors are grateful to Huddersfield University for allowing access to their Talysurf CCI for this research.

\section{References}

[1] Bozic, K. J., Ong, K., Lau, E., Kurtz, S. M., Vail, T. P., Rubash, H. E. and Berry, D. J., "Risk of Complication and Revision Total Hip Arthroplasty Among Medicare Patients with Different Bearing Surfaces," Clin. Orthop. Relat. Res., 468, 9, 2010, 2357-2362.

[2] Macfarlane, J., “10,000 Hip Replacement Patients Told Their Operations May Need to be Reversed after Receiving Faulty Implants," Daily Mail Online, UK, 2010.

[3] Smith, A. J., Dieppe, P., Porter, M. and Blom, A. W., "Risk of Cancer in First Seven Years after Metal-on-Metal Hip Replacement Compared with Other Bearings and General Population: Linkage Study between the National Joint Registry of England and Wales and Hospital Episode Statistics,” Brit. Med. J., 344, 2012, e2383-1-11.

[4] Garrido, A. H., González, R., Cadenas, M. and Battez, A. H., "Tribological Behavior of Laser-Textured NiCrBSi Coatings," Wear, 271, 5-6, 2011, 925-933.

[5] Huang, W., Jiang, L., Zhou, C. X. and Wang, X. L., "The Lubricant Retaining Effect of Micro-Dimples on the Sliding Surface of PDMS," Tribol. Int., 52, 2012, 87-93.

[6] Sakata, F. Y., Santo, A. M. E., Miyakawa, W., Riva, R. and Lima, M. S. F., "Influence of Laser Surface Texturing on Surface Microstructure and Mechanical Properties of Adhesive Joined Steel sheets," Surf. Eng., 25, 3, 2009, 180-186.
[7] Pawlus, P., Cieslak, T. and Mathia, T., "The Study of Cylinder Liner Plateau Honing Process," J. Mater. Process Tech., 209, 20, 2009, 6078-6086.

[8] Mezghani, S., Demirci, I., Zahouani, H. and Mansori, M. E., "The Effect of Groove Texture Patterns on Piston-Ring Pack Friction," Precis. Eng., 36, 2, 2012, 210-217.

[9] Stachowiak, G. W. and Batchelor, A. W., "Engineering Tribology", Butterworth-Heinemann Publication, 2001.

[10] Krupka, I., Hartl, M., Zimmerman, M., Houska, P. and Jang, S., "Effect of Surface Texturing on Elastohydrodynamically Lubricated Contact under Transient Speed Conditions," Tribol. Int., 44, 10, 2011, 1144-1150.

[11] Katta, J., Jin, Z., Ingham, E. and Fisher, J., "Biotribology of Articular Cartilage-A Review of the Recent Advances," Med. Eng. Phys., 30, 10, 2008, 1349-1363.

[12] Yagi, K., Takedomi, W., Tanaka, H. and Sugimura, J., "Improvement of Lubrication Performance by Micro Pit Surfaces,” Tribology Online, 3, 5, 2008, 285-288.

[13] Gao, L., Yang, P., Dymond, I., Fisher, J. and Jin, Z., "Effect of Surface Texturing on the Elastohydrodynamic Lubrication Analysis of Metal-on-Metal Hip Implants,” Tribol. Int., 43, 10, 2010, 1851-1860.

[14] Ito, H., Kaneda, K., Yuhta, T., Nishimura, I., Yasuda, K. and Matsuno, T., "Reduction of Polyethylene Wear by Concave Dimples on the Frictional Surface in Artificial Hip Joints," J. Arthroplasty, 15, 3, 2000, 332-338.

[15] Dumbleton, J. H. and Manley, M. T., "Metal-on-Metal Total Hip Replacement: What does the Literature Say?", J. Arthroplasty, 20, 2, 2005, 174-188.

[16] Dowson, D., Hardaker, C., Flett, M. and Isaac, H. G., " Hip Joint Simulator Study of the Performance of Metal-on-Metal Joints, Part I: The Role of Materials," J. Arthroplasty, 19, 8, 2004, 118-123.

[17] Dowson, D., Hardaker, C., Flett, M. and Isaac, G. H., "A Hip Joint Simulator Study of the Performance of Metal-on-Metal Joints: Part II: design," J. Arthroplasty, 19, 8, 2004, 124-130.

[18] Bergmann, G., Deuretzbacher, G., Heller, M., Graichen, F., Rohlmann, A., Strauss, J. and Duda, G. N., "Hip Contact Forces and Gait Patterns from Routine Activities," J. Biomech., 34, 7, 2001, 859-871.

[19] Brown, S. A., Zumbrunn, G., Fleury-Olela, F., Preitner, N. and Schibler, U., "Rhythms of Mammalian Body Temperature Can Sustain Peripheral Circadian Clocks", Current Biology, 12, 18, 2002, 1574-1583.

[20] Rahmani, R., Shirvani, A. and Shirvani, H., "Optimization of Partially Textured Parallel Thrust 
Bearings with Square-Shaped Micro-Dimple," Tribol. Trans, 50, 3, 2007, 401-406.

[21] Rahmani, R., "An Investigation into Analysis and Optimization of Textured Slider Bearing with Application in Piston-Ring/Cylinder Contact," Ph.D. Thesis, Anglia Ruskin University, Chelmsford, UK, 2008.

[22] Scholes, C. S., Unsworth, A., Hall, M. R. and Scott, R., "The Effects of Material Combination and Lubricant on the Friction of Total Hip Prostheses," Wear, 241, 2, 2000, 209-213.

[23] Choudhury, D., "Wear Reduction in Metal-on-Metal Hip Replacement," Ph.D. Thesis, Anglia Ruskin University, Chelmsford, UK, 2011.

[24] Gandhe, A. and Grover, M., "(i) Head Size, does it Matter?," Current Orthopedics, 22, 3, 2008, 155-164.

[25] Hyperphysics,

Online, http://hyperphysics.phy-astr.gsu.edu/hbase/frict.ht $\mathrm{ml}$ \#coe, Accessed 25/02/2012.

[26] Martin, H. M., "Lubrication of Gear Teeth," Engineering, 102, 1916, 119-121.

[27] Mischler, S. and Muñoz, A. I., "Wear of CoCrMo Alloys Used in Metal-on-Metal Hip Joints: A Tribocorrosion Appraisal," Wear, 297, 1-2, 2013, 1081-1094.

[28] Chiba, A., Kumagai, K., Nomura, N. and Miyakawa, S., "Pin-on-Disk Wear Behavior in a Like-on-Like Configuration in a Biological Environment of High Carbon Cast and Low Carbon Forged Co-29Cr-6Mo Alloys," Acta Materialia, 55, 4, 2007, 1309-1318.

[29] Sawano, H., Warisawa, S. and Ishihara, S., "Study on Long Life of Artificial Joints by Investigating Optimal Sliding Surface Geometry for Improvement in Wear Resistance," Precis. Eng., 33, 4, 2009, 492-498.

[30] Oliveira, A. L. L., Lima, R. G., Cueva, E. G. and Queiroz, R. D., "Comparative Analysis of Surface Wear from Total Hip Prostheses Tested on a Mechanical Simulator According to Standards ISO 14242-1 and ISO 14242-3," Wear, 271, 9-10, 2011, 2340-2345.

[31] Tiainen, V. M., "Amorphous Carbon as a Bio-Mechanical Coating Mechanical Properties and Biological Applications," Diam. Relat. Mater., 10, 2, 2001, 153-160.

[32] Mattei, L., Puccio, F. D., Piccigallo, B. and Ciulli, E., "Lubrication and Wear Modelling of Artificial Hip Joints: A Review," Tribol. Int., 44, 5, 2011, 532-549.

[33] Johnson, K. L., "Contact Mechanics,” Cambridge University Press; Reprint edition, 1987.

\section{Appendix}

The Hertz contact between two cylinders (Johnson,
1987 [33]):

Maximum contact pressure, $P_{\max }=\frac{1 F}{\pi b L}$

Where Half width $b=\sqrt{\frac{4 F\left[\frac{1-v_{1}^{2}}{E_{1}}+\frac{1-v_{2}^{2}}{E_{2}}\right]}{\pi L\left(\frac{1}{R_{1}}+\frac{1}{R_{2}}\right)}}$

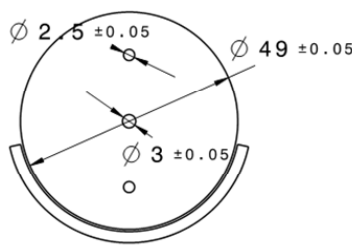

Bottom view Scale: $1: 1$

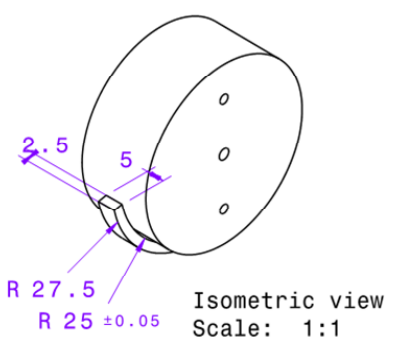

R $25 \pm 0.05$ Scale: $1: 1$
Appendix Fig. 1 The geometry of experiment specimen

For present study the specimen is appeared as:

From the figure 1, and for the material of CrCoMo (F75 allows), the parameter can be defined as

Poisson's ratio, $\gamma=0.3$ (Scholes et al., 2000[22])

Modulus of elasticity, $E_{1} \& E_{2}=2.3 \times 10^{11} \mathrm{~Pa}$ (Scholes et al., 2000)

Applied load for medium speed walking, $F 150 \mathrm{~N}$

Radius of Moving (Upper) specimen $R_{1}=24.5 \mathrm{~mm}$

Radius of fixed specimen (Lower) specimen $R_{2}=-25$ $\mathrm{mm}$

Putting these values in the equation 1 , The half width $b$ is calculated as

$$
\begin{aligned}
b & =\sqrt{\frac{4 F\left[\frac{1-v_{1}^{2}}{E_{1}}+\frac{1-v_{2}^{2}}{E_{2}}\right]}{\pi L\left(\frac{1}{R_{1}}+\frac{1}{R_{2}}\right)}} \\
& =\sqrt{\frac{4 \times 150\left[\frac{1-0.3^{2}}{2.3 \times 10^{11}}+\frac{1-0.32}{2.3 \times 10^{11}}\right]}{3.14 \times 5 \times 10^{-3}\left(\frac{1}{24.5 \times 10^{-3}}+\frac{1}{25 \times 10^{-3}}\right)}} \\
& =6.08 \times 10^{-4} \mathrm{~m} \\
P_{\max } & =\frac{1 F}{\pi b L}
\end{aligned}
$$

Maximum contact pressure,

$$
\begin{aligned}
& =\frac{2 \times 150}{3.14 \times 6.08 \times 10^{-4} \times 5 \times 10^{-3}} \\
& =31.4 \mathrm{MPa}
\end{aligned}
$$

